LEADING ARTICLE

\title{
Mucosal prolapse in the pathogenesis of Peutz-Jeghers polyposis
}

\author{
M Jansen, W W J de Leng, A F Baas, H Myoshi, L Mathus-Vliegen, M M Taketo, H Clevers, \\ F M Giardiello, G J A Offerhaus
}

Gut 2006;55:1-5. doi: 10.1136/gut.2005.069062

Germline mutations in $L K B 1$ cause the rare cancer prone disorder Peutz-Jeghers syndrome (PJS). Gastrointestinal hamartomatous polyps constitute the major phenotypic trait in PJS. Hamartomatous polyps arising in PJS patients are generally considered to lack premalignant potential although rare neoplastic changes in these polyps and an increased gastrointestinal cancer risk in PJS are well documented. These conflicting observations are resolved in the current hypothesis by providing a unifying explanation for these contrasting features of PJS polyposis. We postulate that a genetic predisposition to epithelial prolapse underlies the formation of the polyps associated with PJS. Conventional sporadic adenomas arising in PJS patients will similarly show mucosal prolapse and carry the associated histological features.

See end of article for authors' affiliations

......................

Correspondence to: Dr G J A Offerhaus, Academic Medical Centre of the University of Amsterdam, Meibergdreef 9, 1105 AZ Amsterdam, the Netherlands; g.j.offerhaus@amc.uva.nl

Revised version received 26 July 2005

Accepted for publication

2 August 2005

\section{SUMMARY}

Germline mutations in $L K B I$ cause the rare cancer prone disorder Peutz-Jeghers syndrome (PJS). LKBl can induce complete cellular polarity in single intestinal epithelial cells. Gastrointestinal hamartomatous polyps constitute the major phenotypic trait in PJS. Hamartomatous polyps arising in PJS patients are generally considered to lack premalignant potential although rare neoplastic changes in these polyps and an increased gastrointestinal cancer risk in PJS are well documented. These conflicting observations on the premalignant potential of PJS polyps frustrate the development of evidence based cancer surveillance strategies in PJS patients. The current hypothesis resolves this dilemma by providing a unifying explanation for these contrasting features of PJS polyposis. Several manifestations of PJS, including histopathology of the polyps, are consistent with mucosal prolapse. Analysis of model systems designed to investigate the function of either LKBl or the LKBI interacting protein Parl and its mammalian homologues demonstrates that perturbation of either protein results in consistent phenotypes of epithelial prolapse. We therefore postulate that a genetic predisposition to epithelial prolapse underlies the formation of the polyps associated with PJS. Conventional sporadic adenomas arising in PJS patients will similarly show mucosal prolapse and carry the associated histological features. This notion resolves a persistent dichotomy on the origin of malignant change in PJS polyposis and shifts the focus away from the hamartomatous polyp as the chief precancerous feature in PJS.

\section{INTRODUCTION}

Historically, analysis of familial cancer prone conditions has contributed greatly to the understanding of tumorigenic processes operating in a sporadic setting. PJS is an autosomal dominant hamartomatous polyposis syndrome characterised phenotypically by mucocutaneous pigmentation and the occurrence of hamartomatous polyps throughout the gastrointestinal tract. ${ }^{12}$ These patients have a well established increased cancer risk and many die from malignancies at relatively young age (that is, fifth to sixth decade of life). ${ }^{3}$ In PJS the spectrum of tumours is diverse, and particularly uncommon types of neoplasms outside the gastrointestinal tract can occur. Currently advised screening strategies involve upper endoscopy, colonoscopy, and small bowel series in order to remove gastrointestinal polyps in patients aged 12 years and upwards. ${ }^{4}$ Although gastrointestinal cancers develop in PJS, it remains unclear whether these neoplasms are related to the polyps and whether the polyps carry an inherent (pre)malignant potential.

\section{"Although gastrointestinal cancers develop in PJS, it remains unclear whether these neo- plasms are related to the polyps and whether the polyps carry an inherent (pre)malignant potential $^{\prime \prime}$}

The nature of polyp formation in PJS is unknown. Identification of germline mutations in the $L K B I$ gene, ${ }^{5}$ as the genetic cause in the majority of PJS families, should provide clues about the pathogenesis of PJS polyposis. The $L K B 1$ gene was mapped after the observation of allelic loss of its wild-type counterpart in a minority of polyps from one patient and subsequent linkage of this locus to PJS. ${ }^{5}$ The observation of allelic loss suggested that inactivation of the wild-type $L K B 1$ allele is responsible for the clinical manifestations of PJS, most notably the polyposis phenotype. The frequency of loss of the wild-type allele (loss of heterozygosity $(\mathrm{LOH})$ ) in human PJS polyps remains unclear as the relatively high rate reported in the

Abbreviations: $\mathrm{LOH}$, loss of heterozygosity; PJS, PeutzJeghers syndrome 
literature ${ }^{67}$ may be due in part to induced losses because of technical shortcomings of genetic analyses. Mouse models have shown that haploinsufficiency is sufficient for polyp development ${ }^{8-11}$ and, therefore, a second hit is not a prerequisite for polyp formation (fig lA). One model reported LOH in a minority of murine polyps ${ }^{11}$ but this may have been secondary to, for example, infection. Nevertheless, observations on LOH rates in human PJS polyps implied that loss of a given tumour suppressor function could explain polyp formation. Moreover, the rare observation of adenomatous and carcinomatous change within hamartomatous polyps ${ }^{12-15}$ was taken as proof of a tumorigenic sequence parallel to the adenoma-carcinoma sequence first documented in familial adenomatous polyposis. Hence the concept of a so-called "hamartoma-carcinoma sequence" was born, ${ }^{1617}$ despite limited evidence and the observation that the microscopy of these hamartomas does not resemble (pre)neoplastic growth.

\section{MICROSCOPIC CONSIDERATIONS}

The histopathology of PJS polyps is distinct. Most are pedunculated with a mucosal surface that comprises the epithelial components typical for that particular site of the gastrointestinal tract. The crypts or pits as well as the deeper glands are often elongated, and the mucosa is typically draped on a core of arborising muscle bundles extending from the stalk into the head of the polyp (fig $1 B, C$ ). Although distinctive, this microscopy is not completely specific for PJS polyps. The pathologist can only confidently diagnose a PJS polyp if the patient has PJS or comes from a well established PJS family. Otherwise, strictly speaking, the histopathology report should read: polyp consistent with PJS polyp.
Mucosal prolapse is a lesion or phenomenon that is in the differential diagnosis of a PJS polyp. It can occur at different sites and settings in the gastrointestinal tract and raises various rare diagnoses, such as mucosal prolapse syndrome, cloacogenic polyp, and solitary rectal ulcer syndrome. Similar to PJS polyps, these lesions are characterised by a central area of proliferating muscle fibres surrounded by normal or reactive mucosa believed to be secondary to prolapse or protrusion of the mucosa into the lumen of the gastrointestinal tract (fig 1D, E, F).

"Mucosal prolapse is a lesion or phenomenon that is in the differential diagnosis of a PJS polyp"

Another feature observed as a result of mucosal prolapse is epithelial misplacement into the stalk and submucosa and, interestingly, this is also a common finding in PJS polyps. ${ }^{18}$ This "pseudoinvasion" is due to mechanical forces acting on a polyp during gut motility, which may lead to mucosal herniation into the stalk or submucosa. This finding can cause a spurious diagnosis of malignancy, which may account for a number of cancers reported in PJS polyps. Microscopic similarities between mucosal prolapse and PJS polyps are also pertinent as many PJS patients first come to medical attention with blood loss and symptoms of invagination such as colicky bowel pain or intussusception, features indicative of mucosal prolapse.

\section{GENETIC CONSIDERATIONS}

Recently, it was shown that LKBl induces cell polarity. ${ }^{19} 20$ Loss of cell polarity is a well established feature of neoplastic growth. Moreover, evidence suggests that loss of polarity is
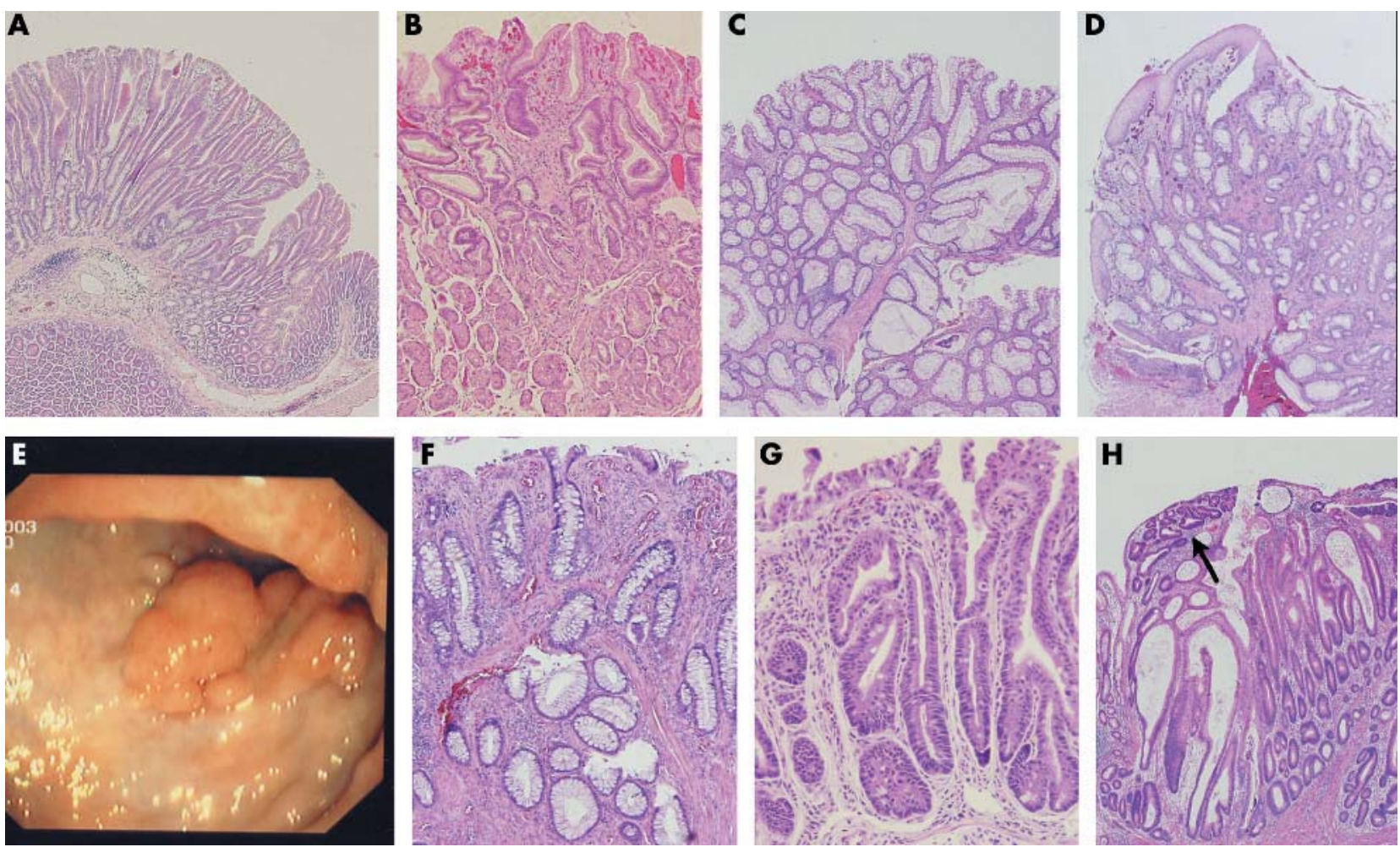

Figure 1 (A) Early gastric polyp in an Lkb1 +/- mouse, aged 29 weeks (Dr Mark Taketo). (B) Early gastric polyp in a patient with Peutz-Jeghers syndrome (PJS) (Johns Hopkins Medical Institution). (C) Early colonic polyp in a PJS patient (Academic Medical Centre). (D) Cloacogenic polyp (Academic Medical Centre). (E) Colonoscopy of a patient initially erroneously considered as PJS and reclassified as mucosal prolapse syndrome (LKB 1 is wild-type in the germline). (F) Microscopy of the colonic polyp from (E). (G) Mucosal prolapse in the colon of a Mark2 - /- mouse (Dr PiwnicaWorms). (H) Adenoma with focally high grade dysplasia (arrow) and features of mucosal prolapse. 
not a consequence but rather a discrete step in the tumorigenic process. ${ }^{21-23}$ This latter consideration begs the question as to whether the role of LKBI in regulating cell polarity can explain the pathogenesis of PJS polyposis and by extension the tumour prone condition.

$L K B 1$, and its evolutionary orthologue par4, belong to a family of PAR genes which constitute a genetic conserved core module that cooperatively directs the execution of a cellular polarity programme. ${ }^{24}$ Recent data from various groups convincingly show that the LKBl and PARl proteins cooperate closely and constitute a kinase cascade in vivo. ${ }^{20}{ }^{25-27}$ Consequently, the results of PARl manipulation in model systems are pertinent, potentially reflecting the effects of disruption of the LKBI/PARl polarity pathway. In mammalian systems, four homologues of the Drosophila and $C$ elegans PARI constitute the family of MARK kinases (that is, MARKl, MARK2, MARK3, and MARK4). Transgenic model systems mutant for PARl/MARK or PAR4/LKBl have generated interesting data regarding the above reasoning on epithelial prolapse in PJS. In C elegans, a phenotype of vulvar prolapse was observed on PARl depletion ${ }^{28}$; in the follicular epithelium of Drosophila oocytes mutant for either parl or $l k b l$, cells are displaced from their adjacent environment ${ }^{29}$; and in murine models of Mark2 knockouts and heterozygotes, established separately in the laboratories of Dr Darmon and Dr PiwnicaWorms, mutant mice present with an unexplained phenotype of colorectal prolapse, ${ }^{3031}$ a lesion similar to a common presenting symptom in PJS patients (fig lG). ${ }^{2}$ These observations of epithelial prolapse have been confirmed in mammalian cell culture systems designed to investigate polarity maintenance where, similarly, cells mutant for MARK2 were squeezed out of an epithelial monolayer. ${ }^{32}$ In conclusion, consistent phenotypes of epithelial and mucosal prolapse have been observed on disruption of either PAR4/LKBl or PARl/ MARK in various model systems, in keeping with close functional and genetic interactions between these gene products.

The PARI/MARK kinases are part of a larger kinase family of AMPK-like kinases.. ${ }^{33}$ This latter family consists of 13 closely related members, including MARKl through MARK4 and the AMP activated protein kinase (AMPK). LKBl activates all but one of the 13 AMPK-like kinases in in vitro kinase assays, underscoring the biochemical conservation of this interaction between LKBl and the AMPK-like kinases. ${ }^{26}$ Two recent studies have expanded on this link, showing that LKBl inhibits mTor by activating AMPK. ${ }^{34}$ Signalling through mTor acts as a cellular switch regulating cellular proliferation with changing nutrient availability. mTor signalling is inhibited under conditions of low nutrients, such as low intracellular ATP levels. Following AMPK activation, mTor inhibition restricts ATP consuming anabolic processes such as protein translation and cell growth in favour of energy conservation. The data in the two aforementioned studies show that mTor activity is regulated by LKB1 through TSC2 after direct activation of AMPK. ${ }^{34}{ }^{35}$ As TSC2 is the tumour suppressor mutated in the tuberous sclerosis complex and mTor activity is further regulated by PTEN, the tumour suppressor mutated in Cowden's disease, the authors converge on the conclusion that three hamartoma syndromes (that is, PJS, tuberous sclerosis complex, and Cowden's disease) are all characterised by dysregulation of mTor activity. ${ }^{34} 35$

Importantly, all biochemical data in these latter studies were gathered in LKBl deficient cells from $L k b l \mathrm{KO}$ embryos. This is relevant as biochemical data are extrapolated to PJS polyps which, as mentioned above, are haploinsufficient with respect to LKBI for polyp formation. This implies that the effects of $L K B I$ heterozygosity on mTor signalling-in contrast with LKBI nullizygosity-have yet to be firmly established. Moreover, from a clinical perspective, other than the word hamartoma there are few similarities between these syndromes, making it appear artificial to group these hamartoma syndromes together. Cowden syndrome is characterised by an increased risk for breast and thyroid cancer. The classical hamartoma in this syndrome is a skin lesion (that is, trichilemmoma). Although these patients may have gastrointestinal polyps, their morphology is different from PJS and there is no gastrointestinal cancer risk. ${ }^{36}$ In tuberous sclerosis complex the lesions typically affect the brain and skin, less frequently hamartomatous abnormalities can be found in the heart, lung, and kidneys, but gastrointestinal manifestations are extremely rare and there is no increased gastrointestinal cancer risk. ${ }^{37}$ Lastly, biallelic deletion of either one of both AMPK isoforms in murine models produces mice which display none of the gastrointestinal effects displayed by $L k b l$ heterozygous or Mark2 heterozygous and deficient mice. ${ }^{38}{ }^{39}$ Furthermore, although AMPK knockout mice show signs of impaired glucose tolerance in line with the presumed role of AMPK as a sensor of cellular energy status, none of these endocrine effects has been associated with PJS. This experimental outcome is in stark contrast with the consistent phenotypes independently described from manipulation of the LKBI/ MARK pathway. To date, the only studies reliably documenting genetic interactions between LKBI and a postulated target in vivo are those between LKBl and PARl/ MARK. ${ }^{20} 2425$

\section{TESTING THE HYPOTHESIS}

The above analysis of consistent phenotypes that arise from mutations in either PAR4/LKBl or PARl/MARK provides a strong impetus to elaborate on the concept of epithelial prolapse underlying PJS polyposis. One can envision how collections of cells squeezed out of their direct environment initiate mucosal prolapse in the gut. Once the prolapse has acquired the size and configuration of a polyp, patients run the risk of invagination. Interestingly, polyp formation in other organs such as the nasopharynx/respiratory tract, gall bladder, and bladder are also reported in PJS, ${ }^{17} 4041$ indicating a general pathophysiological mechanism operative in this disorder.

\footnotetext{
"Interestingly, polyp formation in other organs such as the nasopharynx/respiratory tract, gall bladder, and bladder are also reported in PJS, indicating a general pathophysiological mechanism operative in this disorder"
}

As the present hypothesis departs from the canonical view of polyp formation whereby accumulating genetic accidents drive polypogenesis, genetic alterations obligate for polyp formation will not be demonstrable in hamartomatous PJS polyps. The observed "haploinsufficiency" for polyp formation in murine models ${ }^{8-10}$ agrees with this concept. In fact, only close scrutiny of polyp formation and visualisation of epithelial prolapse in more sophisticated model systems will allow this hypothesis to be tested directly. In view of their transparency, zebra fish could encompass the ideal model system for these studies, providing repeated longitudinal in vivo analysis of target organs. Contributory evidence would entail demonstration of polyclonality of PJS polyps. This coupled with the fact that PJS polyposis has been described on several occasions in newborns ${ }^{42} 43$ argues against accumulation of genetic insults mediating polyp formation. Finally, the current hypothesis predicts that the other phenotypic hallmark in PJS (that is, the mucocutaneous pigmentation) is due to a germline disruption of a polarity regulating pathway. 
FAP
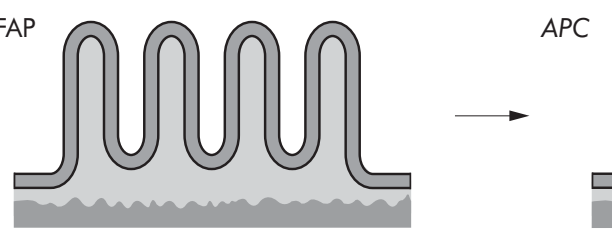

$J P$
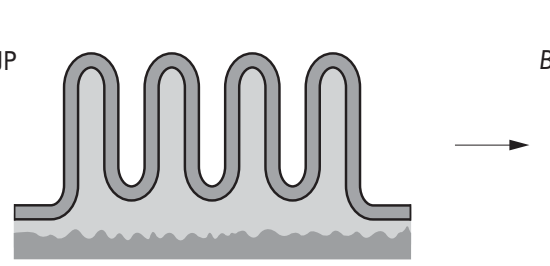

$B M P$
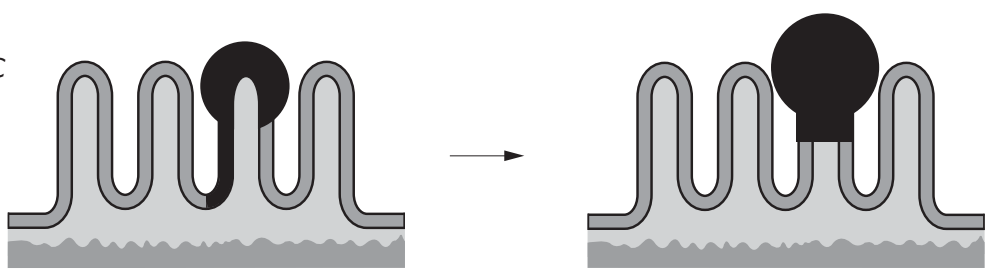

PJS
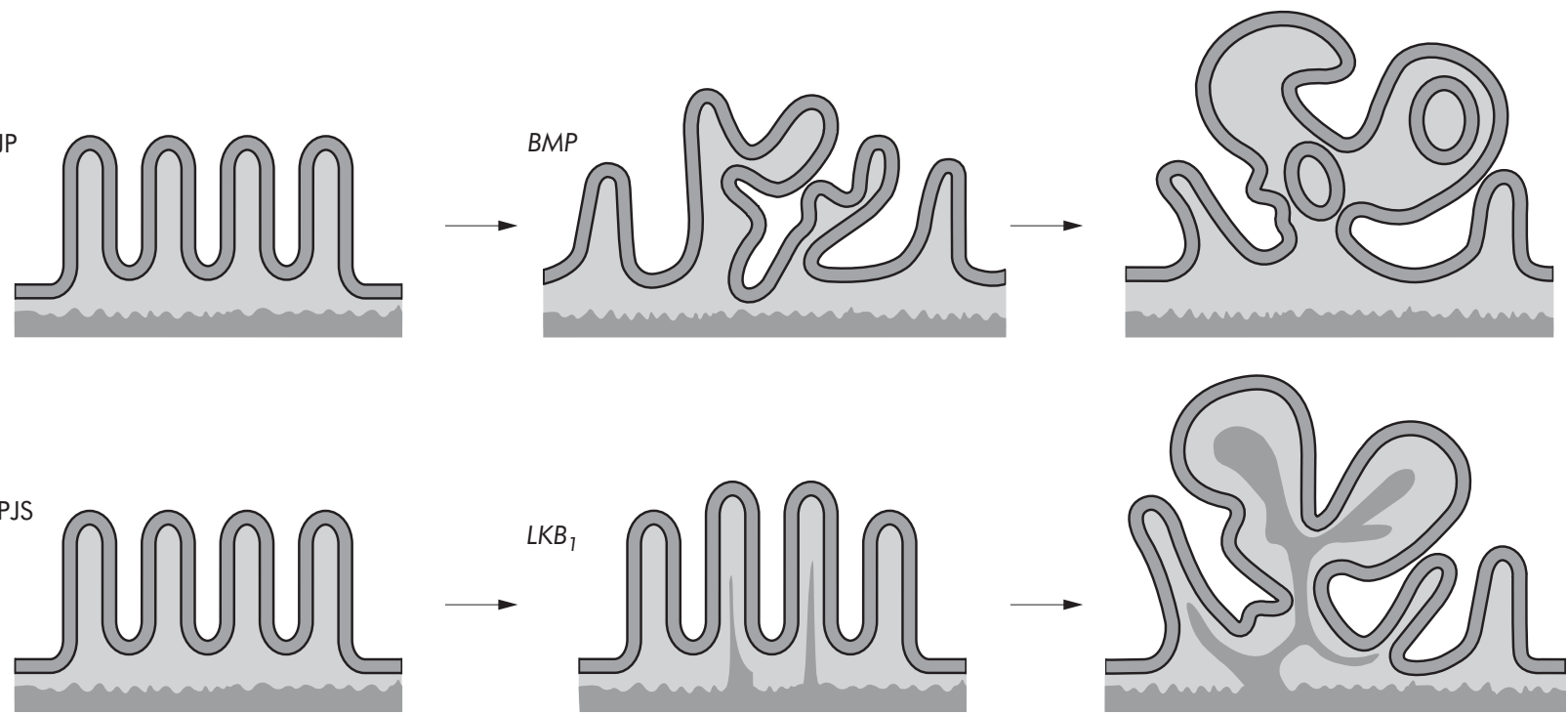

Figure 2 Of the gastrointestinal polyposis syndromes, familial adenomatous polyposis (FAP), and the hamartomatous polyposis syndromes juvenile polyposis (JP) and Peutz-Jeghers syndrome (PJS) are the three main types. In all three syndromes the occurrence of multiple gastrointestinal polyps constitutes the phenotypic hallmark. In FAP disruption of the tumour suppressor gene APC leads to activation of the Wnt pathway, neoplastic proliferation, and clonal, expansion resulting in the formation of adenomas (upper panel). In JP, disruption of BMP signalling due to germline mutations in Smad4 or BMPRIA leads to a landscaper defect and crypts start to bud off or they grow perpendicular to the crypt-villus axis, resulting in the architectural changes typical for those observed in the hamartomatous polyps of JP (middle panel) (see Haramis and colleagues ${ }^{44}$ ). In PJS, a germline mutation of $L K B I$ is associated with loss of cell polarity function. This ultimately leads to epithelial misplacement and secondary changes due to mucosal prolapse (smooth muscle arborisation, pseudoinvasion) and formation of the "hamartomatous" polyps typical of PJS (lower panel). The polyps are non-neoplastic and therefore the hamartoma-carcinoma sequence does not exist (this manuscript).

\section{A UNIFYING CONCEPT OF PJS POLYPOSIS}

As a consequence of the general predisposition to mucosal prolapse in PJS patients, other irregularities in the intestinal mucosal lining, most notably microadenomas, will similarly be prone to mucosal prolapse. Prolapse of incipient adenomas will then result in a histology of dysplasia in a PJS polyp. Microscopic features of mucosal prolapse are regularly observed in conventional adenomatous polyps or carcinomas arisen in an adenomatous polyp (fig $1 \mathrm{H}$ ). The rare observation of dysplasia or even carcinoma in a supposedly preexistent hamartomatous polyp in combination with the known increased gastrointestinal cancer risk in PJS has led to the spurious notion of a unique "hamartoma-carcinoma sequence" operative in PJS. This misconception in the order of events in the formation of dysplastic PJS polyps has confounded research into cancer pathogenesis in PJS. Although loss of the remaining wild-type allele with complete disruption of LKBI polarity function in the polyps of PJS patients may promote tumour formation accounting for the increased cancer risk, gastrointestinal tumour initiation will still adhere to conventionally driven pathways.

\footnotetext{
"The rare observation of dysplasia or even carcinoma in a supposedly pre-existent hamartomatous polyp in combination with the known increased gastrointestinal cancer risk in PJS has led to the spurious notion of a unique "hamartoma-carcinoma sequence" operative in PJS"
}

Interestingly, recent work on a mouse model of juvenile polyposis, the other gastrointestinal hamartomatous polyposis syndrome, indicated that in juvenile polyps, neoplastic growth also follows an adenoma-carcinoma sequence through Wnt activation (fig 2). ${ }^{44}$ This underscores the concept that colorectal cancers are initiated by disruption of a limited set of genetic pathways. The currently presented concept on the origin of malignant change in PJS polyposis reinforces this fundamental postulate.

\section{IMPLICATIONS OF THE HYPOTHESIS}

We postulate that hamartomatous polyp formation in PJS is the result of mucosal prolapse due to a germline disruption of a polarity pathway. This implies that the hamartomatous polyps observed in PJS are an epiphenomenon to the cancer prone condition and not obligate malignant precursors. The hamartoma-carcinoma sequence, as previously postulated, therefore, does not exist in PJS. If proven that the hamartomatous polyps carry no inherent risk of malignancy, our hypothesis bears immediate clinical relevance affecting surveillance strategies in PJS patients. PJS is the first human cancer prone condition in which the polyposis phenotype is linked to a germline disruption of a polarity pathway.

"The hamartomatous polyps observed in PJS are an epiphenomenon to the cancer prone condition and not obligate malignant precursors. The hamartomacarcinoma sequence does not exist in PJS" 
Note added in proof: It has recently been suggested that loss of polarity of function may lead to expansion of the stem cell pool by affecting asymmetric cell division. ${ }^{45}$ It is our belief that expansion of the stem cell compartment accompanied by mucosal prolapse may also be critical for the polyp formation in Peutz-Jeghers Syndrome and it can in addition contribute to cancer predisposition.

\section{ACKNOWLEDGEMENTS}

We thank Drs John Yardley, Guido Tytgat, Belur Bhagavan, Paul Drillenburg, and Anna-Pavlina Haramis for insightful discussions and critique, Drs Michel Darmon and Helen Piwnica-Worms for sharing information and material on unique mouse models, and Drs Josbert Keller and Ralph Carvalho for critically reading and discussing the manuscript.

Supported by a grant from the Netherlands Digestive Disease Foundation (MLDS \#WS 01-03).

\section{Authors' affiliations}

M Jansen, W W J de Leng, L Mathus-Vliegen, G J A Offerhaus, Departments of Pathology and Gastroenterology, Academic Medical Centre, Amsterdam, the Netherlands

A F Baas, H Clevers, Hubrecht Laboratory, Centre for Biomedical Genetics, Utrecht, the Netherlands

H Myoshi, M M Taketo, Department of Pharmacology, Graduate School of Medicine, Kyoto University, Kyoto, Japan

F M Giardiello, Department of Medicine, The Johns Hopkins School of Medicine, Baltimore, Maryland, USA

Conflict of interest: None declared.

\section{REFERENCES}

1 Giardiello FM, Welsh SB, Hamilton SR, et al. Increased risk of cancer in the Peutz-Jeghers syndrome. N Engl J Med 1987;316:1511-14.

2 Westerman AM, Entius MM, de Baar E, et al. Peutz-Jeghers syndrome: 78 year follow-up of the original family. Lancet 1999;353:1211-15.

3 Giardiello FM, Brensinger JD, Tersmette AC, et al. Very high risk of cancer in familial Peutz-Jeghers syndrome. Gastroenterology 2000;1 19:1447-53.

$4 \mathrm{McGrath}$ DR, Spigelman AD. Preventive measures in Peutz-Jeghers syndrome. Fam Cancer 2001;1:121-5.

5 Hemminki A, Markie D, Tomlinson I, et al. A serine/threonine kinase gene defective in Peutz-Jeghers syndrome. Nature 1998;391:184-7.

6 Wang ZJ, Ellis I, Zauber P, et al. Allelic imbalance at the LKBI (STK11) locus in tumours from patients with Peutz-Jeghers' syndrome provides evidence for a hamartoma-(adenoma)-carcinoma sequence. J Pathol 1999;188:9-13.

7 Gruber SB, Entius MM, Petersen GM, et al. Pathogenesis of adenocarcinoma in Peutz-Jeghers syndrome. Cancer Res 1998;58:5267-70.

8 Miyoshi H, Nakau M, Ishikawa TO, et al. Gastrointestinal hamartomatous polyposis in Lkb1 heterozygous knockout mice. Cancer Res 2002;62:2261-6.

9 Rossi DJ, Ylikorkala A, Korsisaari N, et al. Induction of cyclooxygenase-2 in a mouse model of Peutz-Jeghers polyposis. Proc Natl Acad Sci U S A 2002;99:12327-32

10 Jishage K, Nezu J, Kawase Y, et al. Role of Lkbl, the causative gene of PeutzJegher's syndrome, in embryogenesis and polyposis. Proc Natl Acad Sci U S A 2002;99:8903-8.

11 Bardeesy N, Sinha M, Hezel AF, et al. Loss of the Lkbl tumour suppressor provokes intestinal polyposis but resistance to transformation. Nature 2002;419:162-7.

12 Papaioannou A, Critselis A. Malignant changes in Peutz-Jeghers syndrome. N Engl J Med 1973;289:694.

13 Perzin KH, Bridge MF. Adenomatous and carcinomatous changes in hamartomatous polyps of the small intestine (Peutz-Jeghers syndrome): report of a case and review of the literature. Cancer 1982;49:971-83.

14 Hizawa K, lida M, Matsumoto T, et al. Neoplastic transformation arising in Peutz-Jeghers polyposis. Dis Colon Rectum 1993;36:953-7.

15 Defago MR, Higa AL, Campra JL, et al. Carcinoma in situ arising in a gastric hamartomatous polyp in a patient with Peutz-Jeghers syndrome. Endoscopy 1996;28:267
16 Bosman FT. The hamartoma-adenoma-carcinoma sequence. J Pathol 1999;188:1-2.

17 Hemminki A. The molecular basis and clinical aspects of Peutz-Jeghers syndrome. Cell Mol Life Sci 1999;55:735-50.

18 Shepherd NA, Bussey HJ, Jass JR. Epithelial misplacement in Peutz-Jeghers polyps. A diagnostic piffall. Am J Surg Pathol 1987;11:743-9.

19 Baas AF, Kuipers J, van der Wel NN, et al. Complete polarization of single intestinal epithelial cells upon activation of LKB1 by STRAD. Cell 2004; 116:457-66.

20 Martin SG, St Johnston D. A role for Drosophila LKB1 in anterior-posterior axis formation and epithelial polarity. Nature 2003;421:379-84.

21 Pagliarini RA, Xu T. A genetic screen in Drosophila for metastatic behavior. Science 2003;302:1227-31.

22 Brumby AM, Richardson HE. Scribble mutants cooperate with oncogenic Ras or Notch to cause neoplastic overgrowth in Drosophila. Embo J 2003:22:5769-79.

23 Klezovitch O, Fernandez TE, Tapscott SJ, et al. Loss of cell polarity causes severe brain dysplasia in Lgl1 knockout mice. Genes Dev 2004;18:559-71.

24 Macara IG. Parsing the polarity code. Nat Rev Mol Cell Biol 2004;5:220-31.

25 Brajenovic M, Joberty G, Kuster B, et al. Comprehensive proteomic analysis of human Par protein complexes reveals an interconnected protein network. J Biol Chem 2004;279:12804-11.

26 Lizcano JM, Goransson O, Toth R, et al. LKB1 is a master kinase that activates 13 kinases of the AMPK subfamily, including MARK/PAR-1. Embo J 2004; 23:833-43.

27 Spicer J, Rayter S, Young N, et al. Regulation of the Wnt signalling component PARIA by the Peutz-Jeghers syndrome kinase LKBI. Oncogene 2003;22:4752-6.

28 Hurd DD, Kemphues KJ. PAR-1 is required for morphogenesis of the Caenorhabditis elegans vulva. Dev Biol 2003;253:54-65.

29 Doerflinger $\mathrm{H}$, Benton R, Shulman JM, et al. The role of PAR-1 in regulating the polarised microtubule cytoskeleton in the Drosophila follicular epithelium. Development 2003;130:3965-75.

30 Bessone S, Vidal F, Le Bouc Y, et al. EMK protein kinase-null mice: dwarfism and hypofertility associated with alterations in the somatotrope and prolactin pathways. Dev Biol 1999:214:87-101.

31 Hurov JB, Stappenbeck TS, Zmasek CM, et al. Immune system dysfunction and autoimmune disease in mice lacking Emk (Par-1) protein kinase. Mol Cell Biol 2001;21:3206-19.

32 Bohm H, Brinkmann V, Drab M, et al. Mammalian homologues of C. elegans PAR-1 are asymmetrically localized in epithelial cells and may influence their polarity. Curr Biol 1997;7:603-6

33 Baas AF, Smit L, Clevers H. LKB1 tumor suppressor protein: PARtaker in cell polarity. Trends Cell Biol 2004;14:312-19.

34 Corradetti MN, Inoki K, Bardeesy N, et al. Regulation of the TSC pathway by LKB1: evidence of a molecular link between tuberous sclerosis complex and Peutz-Jeghers syndrome. Genes Dev 2004;18:1533-8.

35 Shaw RJ, Bardeesy N, Manning BD, et al. The LKB1 tumor suppressor negatively regulates mTOR signaling. Cancer Cell 2004;6:91-9.

36 Merg A, Howe JR. Genetic conditions associated with intestinal juvenile polyps. Am J Med Genet 2004;129C:44-55.

37 Melean G, Sestini R, Ammannati F, et al. Genetic insights into familial tumors of the nervous system. Am J Med Genet 2004;129C:74-84.

38 Viollet B, Andreelli F, Jorgensen SB, et al. The AMP-activated protein kinase alpha2 catalytic subunit controls whole-body insulin sensitivity. J Clin Invest 2003;111:91-8.

39 Jorgensen SB, Viollet B, Andreelli F, et al. Knockout of the alpha2 but not alpha1 5'-AMP-activated protein kinase isoform abolishes 5-aminoimidazole4-carboxamide-1-beta-4-ribofuranosidebut not contraction-induced glucose uptake in skeletal muscle. J Biol Chem 2004;279:1070-9.

40 Vogel T, Schumacher V, Saleh A, et al. Extraintestinal polyps in Peutz-Jeghers syndrome: presentation of four cases and review of the literature. Deutsche Peutz-Jeghers-Studiengruppe. Int J Colorectal Dis 2000;15:118-23.

41 Keller JJ, Westerman AM, de Rooij FW, et al. Molecular genetic evidence of an association between nasal polyposis and the Peutz-Jeghers syndrome. Ann Intern Med 2002;136:855-6.

42 Fernandez Seara MJ, Martinez Soto MI, Fernandez Lorenzo JR, et al. PeutzJeghers syndrome in a neonate. J Pediatr 1995;126:965-7.

43 Al Faour A, Vrsansky P, Abouassi F, et al. Peutz-Jeghers colonic tumour in a newborn. Eur J Pediatr Surg 2002;12:138-40.

44 Haramis AP, Begthel $H$, van den Born $M$, et al. De novo crypt formation and juvenile polyposis on BMP inhibition in mouse intestine. Science 2004:303:1684-6.

45 Clevers H. Stem cells, asymmetric division and cancer. Nat Genet 2005;37:1027-8

For further information please go to: www.quality.bmipg.com

Book early to benefit from a discounted delegate rate 
might make the handbook marginally more user friendly. It would be easy to recommend this handbook to those wanting a quick entry into the world of meta-analysis. The outputs produced would not be wrong in statistical terms. But unless one wanted to make use of the less conventional outputs (and this would generally only apply to experienced reviewers and analysts anyway), it would make much better long term sense to become familiar with the Cochrane software and the entire systematic review package.

\section{NOTICE}

Joint Meeting: International Association of Pancreatology and American Pancreatic Association

This meeting will be held on 1-4 November 2006 and the CME sponsor is American College of Surgeons. The meeting will be heald at Westin Chicago River North Hotel, Chicago, Illinois, USA. For more information please contact APA Headquarters, 45 High Valley Drive, Chesterfield, MO 63017; http:// www.american-pancreatic-association.org/;

D Forman lettuceplanet.com; teil: +1 314210 2904; fax: +1 3147549515 .

\section{CORRECTION}

doi: 10.1136/gut.2005.08195corr2

In the March supplement to Gut (Gut 2006;55(suppl 1)) the affiliation of the author Dr V Villanacci was provided incorrectly. His correct address is: 2nd Department of Surgical Pathology, Spedali Civilli, Brescia, Italy.

\section{EDITOR'S QUIZ: GI SNAPSHOT}

Answer

From question on page 742

A differential included hydatid cyst and cystic tumour metastasis. The cyst was aspirated under computerised tomography (CT) guidance which showed endometrial glandular and stromal elements confirming a diagnosis of endometrial cyst.

Clinical features are non-specific. Theories behind the pathogenesis include coelomic metaplasia and lymphovascular dissemination. Imagings show a $12 \mathrm{~cm}$ loculated cystic lesion with irregular outline in the posterior aspect of the right lobe of the liver. Ultrasound and CT findings in endometrial liver cysts are non-specific and may include cystic and solid components, septations, loculations, and calcifications. On magnetic resonance imaging, endometrial implants usually demonstrate signal intensity similar to normal endometrium on T1 and T2 weighted images. A CT scan repeated during the menses is valuable in suspected pulmonary endometriosis. ${ }^{1}$ It is unknown if the same approach would benefit diagnosis in suspected hepatic endometrial cysts. Although there is no literature to support or refute, we wonder if repeating a CT scan coincident with menses might help in the diagnosis of a suspected hepatic endometrial cyst. Although surgery is the treatment of choice, gonadotrophin releasing hormone analogues have been used for treating pulmonary endometriosis and may prove beneficial in hepatic endometrial cyst.

Teaching point: This differential should be kept in mind when dealing with a hepatic cyst of unknown origin in a female, especially with history of endometriosis or pelvic surgery.

doi: 10.1136/gut.2005.066498

\section{REFERENCE}

1 Terada Y, Chen F, Shoji T, et al. A case of endobronchial endometriosis treated by subsegmentectomy. Chest 1999;115:1475-8. 